\title{
FLUOROSCOPIC GUIDED LUMBAR INTERLAMINAR EPIDURAL INJECTIONS: A Prospective Evaluation of Epidurography Contrast Patterns and Anatomical ReView of the Epidural Space
}

\author{
Kenneth P. Botwin, MD, James Natalicchio, MD, and Ashraf Hanna, MD
}

\begin{abstract}
Objective: To evaluate the pattern and flow of epidural contrast in fluoroscopically guided lumbar interlaminar steroid injections.

Design and Methods: A prospective case series of 25 (twenty-five) consecutive patients receiving 25 (twenty-five) injections. Patients had either lumbar spinal stenosis (LSS) or herniated nucleus pulposus (HNP). All patients received their injection using a loss of resistance technique. Once the epidural space was felt localized $0.5 \mathrm{~mL}$ of Isovue contrast was injected to confirm accurate needle placement. If in the epidural space, another $4.5 \mathrm{~mL}$ was injected for a total of 5
\end{abstract}

Epidural steroid injections have been used in the treatment of lumbar radicular pain syndrome since 1952 (1).

First reported in the United States in 1960, it was found to benefit conditions causing nerve root irritation (2). These injections were performed "blind" (without fluoroscopic guidance), using an interlaminar "loss of resistance technique." Even in experienced hands, improper localization of the epidural space using the blind technique has occurred in $13 \%$ to $30 \%$ of attempted injections $(3,4)$. Lumbar interlaminar epidural injections have been performed using a blind technique with lack of resistance to air or normal saline (5). Utilizing fluoroscopic technique proper localization of the epidural space can be confirmed (6).

Lutz and Wisneski (7) reviewed 50 patients with lumbar radiculopathy from HNP who responded well to transforaminal epidural steroid injections. They postulated that the delivery of epidural ste-

From Florida Spine Institute, Clearwater, Florida. Address Correspondence: Kenneth P. Botwin, MD, 2250 Drew Street, Clearwater FL-33765.

E-mail: contactus@floridaspineinstitute.com

Funding: There was no external funding in preparation of this manuscript.

Conflict of Interest: None
$\mathrm{mL}$ contrast media. Both AP and lateral radiographs were obtained and reviewed by a physician trained in fluoroscopic injections for review of the contrast pattern. Patterns recorded were Unilateral, Bilateral, Ventral or Dorsal. The dorsal flow was also characterized as being cephalad or caudad and the number of lumbar intervertebral levels of flow were recorded as well.

Results: Dorsal contrast flow occurred in all 25 injections. Thirty-six percent ( 9 out of 25) resulted in ventral spread of contrast. Eighty-four percent (21 out of 25) of the injections had flow of contrast unilaterally and $16 \%$ (4 out of 25 ) was bilateral. The mean number of levels of flow of contrast cephalad from the injection site was 1.28 and caudally 0.88 . There was a significant difference in more cephalad than caudal contrast flow $(P=0.004)$

Conclusion: Thirty six percent of the injections observed in the study revealed ventral contrast flow. Bilateral contrast flow occurred in $16 \%$ of the injections. Caudad contrast flow is less than cephalad. The observed contrast flows need to be studied clinically to determine if this can affect clinical outcome.

roscopy. roid to the intervertebral disc nerve root region in the ventral epidural space leads to a good clinical outcome.

As patients with lumbar radicular pain continue to receive epidural injections in the treatment of the pain syndrome we are interested in determining whether medications administered in the interlaminar approach were spreading into the ventral epidural space as has been described in transforaminal epidural injections (8).

Several studies have demonstrated the degree of epidural spread both vertically and laterally (9-13). However, review of the medical literature revealed no published studies evaluating the spread of solutions into the lumbar epidural space interlaminarly and evaluating the amount of ventral epidural spread.

Thus, we present the first prospective case series evaluating the amount of ventral spread in the epidural space present after fluoroscopically guided lumbar interlaminar epidural injection.

\section{Methods}

Twenty-five patients presented to a multi disciplinary spine care practice with complaints of low back and lumbar radicular pain. As part of a non-operative treatment plan, patients received a combination of anti-inflammatories analgesics, referral to physical therapy and fluoroscopically guided interlaminar lumbar epidural steroid injections.

All epidural injections were performed at an ambulatory surgical center by one of three physicians with extensive experience in fluoroscopically guided lumbar interlaminar epidural injections. A prospective case series of 25 patients who had 25 injections were included in the study. Inclusion criteria consisted of any injection performed in these consecutive patients for the treatment of lumbar radicular pain from either HNP (herniated nucleus pulposus) or lumbar stenosis (LSS). The diagnosis was based upon the clinician performing the procedure noting it on the patients chart. Exclusion criteria consisted of any history of lumbar spinal surgery, allergy to contrast dye, active infection in the lumbar area, any allergy to anesthetic or corticosteroid.

The technique used in all 25 injections was standardized. Patients were placed prone on a radiology table. Their back was prepared using an iodine-based antiseptic solution (a) and an alcohol solution (b). Using a fluoroscope, and an 18ga or 20ga 3.5inch / $90 \mathrm{~mm}$ Tuohy 


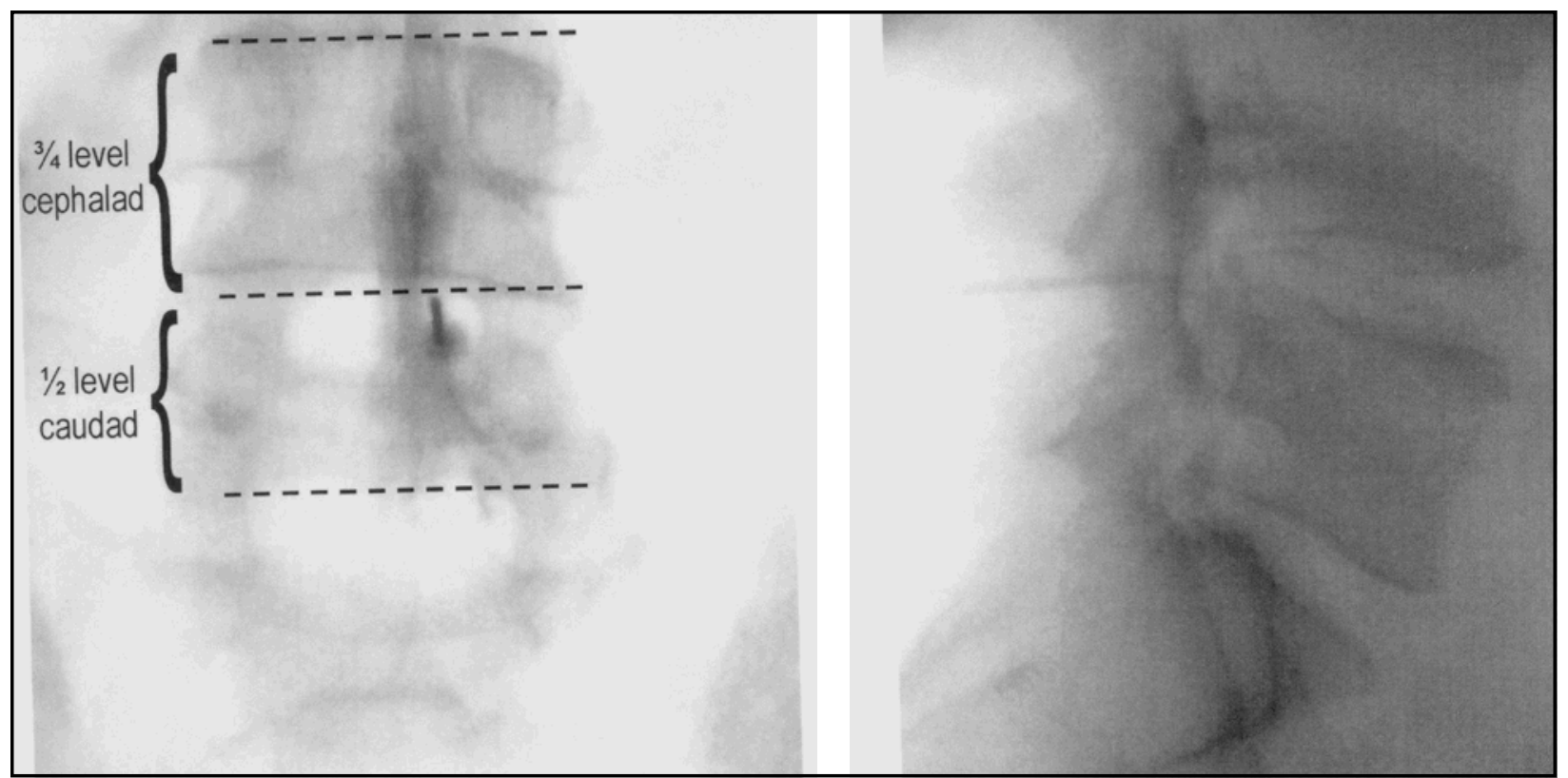

Fig. 1. Anteroposterior radiograph (left) of interlaminar injection at L4-5. Note contrast flow cephalad of 0.75 levels and caudad contrast flow of 0.5 levels. Lateral radiograph (right) reveals contrast flow in dorsal epidural space

spinal needle (c) were guided under intermittent fluoroscopic guidance to the dorsal/ epidural space using loss of resistance technique with normal saline at the L4/5 or L5/S1 level. Once the epidural space was felt localized $0.5 \mathrm{~mL}$ of Isovue contrast was injected to confirm accurate needle placement. An anteroposterior and lateral radiograph was obtained to confirm this accurate needle placement. If in the epidural space another $4.5 \mathrm{~mL}$ of Isovue M-300, Iopamidol injection, (d) was injected for a total of 5 $\mathrm{mL}$ contrast media. Both and AP and lateral radiographics were then obtained by a radiologic technician. The radiologic technician gave all radiographs to an independent physician who was trained in fluoroscopic injections but did not perform the injections on the involved patients. The epidurograms were viewed to evaluate the contrast pattern observed on the epidurograms. Contrast patterns were described as being unilateral, bilateral, ventral and or dorsal. The ventral and dorsal flow was also described as being cephalad or caudad and the number of lumbar intervertebral levels of flow was recorded. A level was de-

a. Povidone lodine USP solution, Clinipad, Rocky Hill CT

b. Kendall Webcol Alcohol Prep 70\% Isopropyl alcohol, Marshfield, MA

c. Becton Dickinson, Franklin Lakes NJ

d. Bracco Diagnostics, Princeton NJ fined as being from the middle of the intervertebral disc from where the injection was performed to the one cephalad or caudad. Thus, in an injection at L4-5 if contrast reached the L3-4 disc it would be recorded as one level of flow cephalad. At the L5-S1 level the caudad level was described to reach the level of the S1 foramina, the levels were rounded off in quarter intervals (Fig. 1). Patients also received $5 \mathrm{~mL}$ of $1 \%$ preservative free Xylocaine and $80 \mathrm{mg}$ Kenalog (Triamcinolone Acetate) following radiographic evaluation.

Statistical analysis was performed to evaluate if there was a significant difference in the levels of contrast flow both caudad and cephalad in the HNP vs LSS patient injections.

Analysis was also performed in the HNP patient injections to see if there was a significant difference in caudad to cephalad contrast flow as well as in the LSS patient injections.

\section{RESULTS}

Our data was obtained from 25 consecutive fluoroscopically guided interlaminar epidural injections on 25 different patients. There were 17 male and 8 female patients. Of these patients, 12 had lumbar spinal stenosis (LSS) and 13 had herniated nucleus pulposus (HNP). The mean age of the stenosis patients was 66 years (4386) and the mean age of the HNP patients was 50.3 years (31-60). The mean height $(\mathrm{cm})$ of the LSS patients was 169.1 (152.4 $-185.4 \mathrm{~cm})$ and in the HNP patients was $176.7 \mathrm{~cm}(153.7-185.4 \mathrm{~cm})$.

Review of all epidurograms in all patients revealed contrast in the dorsal epidural space. There were 9 that had contrast visualized in the ventral epidural space out of the 25 injections (36\%). 4 were in HNP patients and 5 in LSS patients. All these patients had dorsal flow as well.

Review of contrast patterns showed unilateral flow (not crossing the midline from site of injection) in 21 of the 25 injections (84\%). Bilateral contrast pattern was seen in 4 injections (16\%). The injections were performed at different intervertebral levels 18 at L5-S1, 4 at L4-5, 2 at L2-3 and 1 at L3-4.

The mean amount of caudad spread of contrast from the injection site was recorded and found in all 25 injections to be a mean of 0.88 levels. The mean amount of cephalad spread of contrast was 1.28 levels (Table 1). There was a significant difference with more cephalad contrast flow than caudad.

The mean amount of contrast flow cephalad from the injection site in the HNP patient injections was 1.12. The amount of contrast flow caudad in the HNP patient injections was 0.96 . The mean amount of contrast flow cephalad from the injection site in the steno- 
Table 1. Mean levels of contrast flow from injection site in 25 injections (one level defined as being from the middle of the intervertebral disc from where the injection was performed to the one cephalad or caudad)

\begin{tabular}{|c|c|c|c|c|}
\hline & $\begin{array}{c}\text { HNP - INJECTION } \\
(\mathrm{n}=13)\end{array}$ & $\begin{array}{c}\text { LSS - INJECTION } \\
(\mathrm{n}=12)\end{array}$ & $\begin{array}{c}\text { Total } \\
(\mathrm{n}=25)\end{array}$ & $P$ value \\
\hline Caudad & $\begin{array}{c}0.96 \pm 0.43 \\
(0.5-2.0)\end{array}$ & $\begin{array}{c}0.79 \pm 0.26 \\
(0.5-1.0)\end{array}$ & $\begin{array}{c}0.88 \pm 0.36 \\
(0.5-2.0)\end{array}$ & 0.249 \\
\hline Cephalad & $\begin{array}{c}1.12 \pm 0.46 \\
(0.5-2.0)\end{array}$ & $\begin{array}{c}1.46 \pm 0.58 \\
(0.5-2.0)\end{array}$ & $\begin{array}{l}1.28 \pm 0.54 \\
(0.5-2.0))\end{array}$ & 0.120 \\
\hline$P$ value & 0.390 & 0.002 & 0.004 & \\
\hline
\end{tabular}

Values in parenthesis indicate range

sis patient injections was 1.46 . The mean amount of contrast flow caudad in the LSS patient injections was 0.79 . There was no significant difference in mean number of levels of contrast flow comparing caudad flow in HNP to LSS and in cephalad flow in HNP to LSS patient injections (Table 1).

A Review of HNP patient injections was done to determine the amount of caudad and cephalad contrast flow with statistical analysis to see if contrast was either more cephalad or caudad in flow (Table 1). There was more contrast flow cephalad in LSS patient injections.

\section{DisCUSSION}

The history of epidurography dates back to 1921. This first viewing of the epidural space was performed, reportedly by accident, by Sicard and Forestier using Lipoidal as the contrast agent (12).

Using both air and a water-soluble contrast medium, Perabrodil, radiographic examination of the epidural space was again described in $1941(14,15)$.

Metrizamide, a nonionic approved by the FDA for myelography in 1978 was used to visualize the lumbar epidural space in the 1980's. Hatten (11) proposed epidurography is a valuable tool providing clinicians with important anatomical information in evaluation of patients with equivocal myelography and or confusing or non-diagnostic physical findings.

These early studies utilized a caudal approach with catheters rather than a single injection at a specific site $(11,12,14$, 15). Emphasis was placed on evaluation of transverse, caudad and cephalad flow. Minimal attention was given to analyzing the extent of anterior or ventral flow.

More recently, spinal interventionalists have employed a form of epidurography to confirm accuracy of contrast-enhanced fluoroscopically guided selective injections.

Such injections using anesthetic and corticosteroid preparations have been used extensively to treat low back pain and radicular symptoms arising from compressive lesions such as disc herniations and spinal stenosis.

It has been suggested that the efficacy of such injections is dependent upon several factors including:

1. The particular approach employed, i.e., transforaminal vs interlaminar vs caudal. Proponents of the transforaminal approach argue that increased efficacy is obtained by more ventral placement of the injectate near the disc-nerve root interface.

2. The anatomic variation of epidural space.

3. The flow of injectate.

4. The skill of the interventionalist.

5. The type and extent of the pathology.

\section{Anatomy of the Epidural Space}

The epidural space extends from the foramen magnum to the end of the dural sac at S2. The actual size of the posterior epidural space can very greatly. It expands to 5 to $6 \mathrm{~mm}$ at its greatest width in the mid-lumbar spine and gradually decreases to about $2 \mathrm{~mm}$ at the S1 level (16).

The epidural space surrounds the dural sac. It is bordered posteriorly by the ligamentum flavum and periosteum and anteriorly by the posterior longitudinal ligament and vertebral bodies. Laterally it is bordered by the pedicles and intervertebral foramina.

The epidural space is widest in the midline underneath the junction of the lamina and narrows laterally beneath the zygapophyseal joint. Laterally the ligamentum flavum joins with the joint capsule of the adjacent zygapophyseal joint.
The posterior epidural space contains the ligamentum flavum and the dorsomedian connective tissue. The principal content within the epidural space is fat. Also within the epidural space run the internal vertebral venus plexus, the spinal branches of the segmental arteries, lymphatics and the dura arachnoid projections that surround the spinal nerve roots (17).

The ligamentum flavum has been proposed to be joined in the midline. There appears to be a paired nature to the ligament having both a right and left portion $(18,19)$. Cryomicrotome sectioning performed on the epidural space has shown that there is a variable degree of fusion of the ligamentum flavum in the midline (20).

There have been histologic studies showing that the fat in the epidural space is remarkable for its homogenous structure and its lack of fibrous content (21). The actual epidural sac has been shown to separate from the ligamentum flavum and dura except in the midline (20). When a needle is placed in the direct midline area it may actually penetrate through a very thin ligamentum flavum, which would possibly lead clinically to a lack of actual resistance. This may be one of the reported benefits of a paramedian approach compared to a direct midline approach for epidural needle placement (22).

The actual size and shape of the epidural space is determined by the manner of attachment of the dural sac to the walls of the spinal canal. There have been multiple studies that have actually demonstrated a dorsomedian connective tissue band in the lumbar epidural space $(10,23$, 24). The posterior epidural space is divided by the plica mediana dorsalis and additional transverse connective tissue planes (25). There is a compartmentalized nature to the space, which can account for 
limitation of the flow substances.

Of the 25 patient injections we studied, 9 had contrast flow to the ventral epidural space. Thus, $36 \%$ of these injections appeared to reach the ventral epidural space. This may be of importance in clinical outcomes, as it is perceived the inflammatory response tends to be localized at the nerve/root intervertebral disc interface. Eight-four percent of injections appeared to result in unilateral flow of contrast. This may be an important finding as patients with bilateral lower extremity pain syndromes may require bilateral paramedian injections in order to assure a proper delivery of corticosteroid at the target areas. Of course, clinical outcome studies need to be done in order to assess this findings significance clinically.

Statistical analysis of contrast flow patterns in all 25 injections revealed greater cephalad flow than caudad flow. It also revealed in LSS patient injections a statistically significant amount of more cephalad than caudad contrast flow.

Shortcomings of the study are several. The overall number of patients is small and comparing the few numbers of LSS to HNP patients should be done with a larger sample size in order to draw conclusions as to the overall contrast patterns within these individual diagnoses. Higher volumes of injectate may also result in different flow patterns.

We cannot draw any clinical conclusions from this data. More research is needed to validate our observations.

\section{ConcLusion}

Thirty six percent of the injections observed in the study revealed ventral contrast flow. Bilateral contrast flow occurred in $16 \%$ of the injections. Caudad contrast flow is less than cephalad. The observed contrast flows need to be studied clinically to determine if this can affect clinical outcome.

\section{Author Affiliation: \\ Kenneth P. Botwin, MD \\ Fellowship Director \\ Florida Spine Institute \\ 2250 Drew Street \\ Clearwater, FL-33765 \\ E-mail: contactus@floridaspineinst itute.com}

\section{James Natalicchio, MD}

Fellow Interventional Physiatry

Florida Spine Institute

2250 Drew Street

Clearwater, FL 33765

E-mail: contactus@floridaspineinst itute.com

\section{Ashraf Hanna, MD}

Pain Management/ Anesthesia

Florida Spine Institute

2250 Drew Street

Clearwater, FL 33765

E-mail:contactus@floridaspineinsti tute.com

\section{REFERENCES}

1. Robechhi, Capra R. L'Idrocotisone (Composto F), Rine Esperinze Cliniche in Campo Rheumatologico. Minerva MED 1952; 98: 1259-1263.

2. Benzon H. Epidural steroid injections for low back pain and lumbosacral radiculopathy. Pain 1986; 24:277-295.

3. Mehta M. Extradural block. Confirmation of the injection site by $\mathrm{x}$-ray monitoring. Anesthesia 1985; 40:1009-1012.

4. White AH. Injection techniques for the diagnosis and treatment of low back pain. Orthop Clin North Am 1983; 14:553-567.

5. Bromage PR. The "hanging drop" sign. Anesthesia 1953; 8:237-241.

6. Johnson BA, Schellhas KP, Pollei SR. Epidurography and therapeutic epidural injections: Technical considerations and experience with 5334 cases. Am J Neuroradiol 1999; 20:697-705

7. Lutz GE, Wisneski RJ. fluoroscopic transforaminal lumbar epidural steroid injection: Outcome study. Arch Phys Med Rehabil 1998; 79:1362-1366.

8. Andrade A, Eckman E. The Distribution of Radiologic Contrast Media by Lumbar Translaminar and Selective Neural Canals in Normal Human Volunteers. Proceedings of the International Spinal Injection Society Annual Meeting. Keystone (CO), 1992.

9. Burn JM, Guyer PB, Langdon L. The spread of solutions injected into the epidural space. Brit J Anesth 1973; 45:338-345.

10. Blomberg R. The dorsomedian connective tissue band in the lumbar epidural space of humans: An anatomical study using epiduroscopy in autopsy cases. Anesthesia Analg 1986; 65:747-752.

11. Hatten HP. Lumbar epidurography with metrizamide. Radiology 1980; 137:129136.

12. Luyendijk W, vanVoorthuisenae. Contrast examination of the spinal epidural space. Acta Radiol 1966; 5:1051-1066.

13. Roberson GH, Hatten HP, Hesselink JH. Epidurography: Selective catheter techniques and review of 53 cases. Am J Roentgenol 1979; 132:787-793.

14. Sanford H, Doub HP. Epidurography: A method of roentgenologic visualization of protruded intervertebral disks. Radiology 1941; 38:712-718.

15. Knutsson F. Experiences with epidural contrast investigation of the lumbosacral canal in disc prolapse. Acta Radiol 1941; 22:694-703.

16. Cheng PA. The anatomical and clinical aspects of epidural anesthesia. Curr Res Anesth Analg 1963; 42:398-415.

17. Bonica JJ. The Management of Pain. Lea and Febiger, Philadelphia, 1990:1411-1413

18. Ramsey RH. The anatomy of the ligamenta flava. Clin Ortho 1966; 44:129-140.

19. Ho PSP, Yu S, Sether LA et al. Ligamentum flavum: Appearance on sagittal and coronal MR images. Radiology 1988; 168:469-472.

20. Hogan QH. Lumbar epidural anatomy a new look by cryomicrotome/section. Anesthesiology 1991:75:767-775.

21. Ramsey HJ. Fat in the epidural space of young and adult cats. Am J Anat 1959; 104 345-380.

22. Bloomberg R, Joanivald A, Walther S. Advantages of the paramedian approach for lumbar epidural analgesia with catheter technique. Anesthesia 1989; 44:742-746.

23. Blomberg RG, Olsson SS. The lumbar epidural space in patients examined with epiduroscopy. Anesth Analg 1989; 68:157-160.

24. Husemeyer, RP, White DC. Topography of the lumbar epidural space. Anesthesia 1980; 35:7-11.

25. Savolaine ER, Pandya JB, Greenblatt SH et al. Anatomy of the human lumbar epidural space: new insights using ct-epidurog raphy. Anesthesiology 1988; 68:217-220. 